\title{
EDITORIAL
}

\section{El análisis de red en la evaluación dinámica ante el impacto de la COVID-19}

\section{Network analysis in the dynamic assessment of the impact of COVID-19}

Cristian Antony Ramos-Vera (iD) ${ }^{1}$

1. Universidad Cesar Vallejo. Lima, Perú. Correo: cristony_777@hotmail.com - https://orcid.org/0000-0002-3417-5701

Se ha destacado el impacto de las diversas medidas tomadas para mitigar la propagación de la infección del virus de la COVID-19, entre ellas el distanciamiento social, lo cual afianza la recurrencia de trastornos mentales existentes. Una de las reacciones comunes ante estas estrategias es el estrés, que propicia una mayor vulnerabilidad a agravar diversas condiciones de salud comórbidas. Esto plantea desafíos específicos sobre las condiciones de salud ante el impacto de la COVID19, que requiere comprender cómo los mecanismos fisiopatológicos de un individuo interactúan entre sí, dada la muestra de estudio (por ejemplo, trabajadores del sector salud, personas con diagnóstico de enfermedad mental, pacientes con enfermedad crónica, entre otros).

Por lo tanto, para una evaluación más dinámica y de implicancia clínica se expone el análisis de redes. Este método gráfico representa una serie de entidades o nodos, que pueden corresponder a variables, constructos o individuos, ante una mayor difusión del análisis de redes sociales que considera a las personas como unidades de la red ${ }^{1}$. Recientemente, durante la última década fue incluida en el ámbito clínico otra variante del análisis de red, representada por correlaciones estadísticas (dirigidas o no dirigidas) con base en la teoría de grafos $^{2,3}$. Estas asociaciones son de orden cero o relaciones parciales causales que conectan a los nodos (variables) y estructuran el modelo dinámico. En este modelo se incluyen diversas medidas y condiciones clínicas ${ }^{4}$ neuropsicológicas y cognitivas $^{5}$, neuroanatómicas ${ }^{6}$, bioquímicas ${ }^{7}$, genómicas $^{8}$, antropométricas ${ }^{9}$, entre otras. La representación gráfica facilita la interpretación de manera sencilla: mientras más gruesa sea la conexión entre las variables, mayor será la relación estadística ${ }^{2,3}$.

El modelo de red es un análisis multivariante compuesto por múltiples relaciones no lineales regularizadas (eliminación de relaciones más espurias mediante el estimador LASSO) después del control multivariado de los elementos de la red ${ }^{2,3}$. Esto favorece la inclusión de variables de diversa naturaleza para evaluar múltiples aspectos de salud y de la condición comórbida de una persona, así como la interacción entre sus múltiples factores etiológicos y moduladores. Este método se ha incluido en estudios instrumentales que han evaluado la impulsividad, la autoagresión, la ideación suicida ${ }^{10}$ y el crecimiento postraumático en adolescentes $^{11}$.

La aplicación del análisis de red permite estimar los elementos "puente" (altos índices de centralidad) que refieren mayor implicancia clínica, algo que resulta de mayor requerimiento ante el contexto actual debido al impacto psicológico negativo del COVID-19. Aquellos elementos afectan las interacciones de los demás componentes, es decir, una mayor medida de este elemento "puente" aumenta la probabilidad de fortalecer las demás relaciones, y viceversa: es posible que su 
disminución o una menor medida reduzca las demás conexiones e incluso genere un colapso en toda la estructura de la red ${ }^{2,3}$.

Los análisis de redes son primordiales en la planificación y el desarrollo personalizado de programas de tratamiento y psicoterapia más eficaces ante el contexto de la COVID-19. Por ende, se requiere mayor difusión de este método para precisar aquellas variables clínicas más comórbidas que afecten las condiciones o los diagnósticos de salud ante el contexto de la COVID-19.

También es posible comparar dos estructuras de red, antes y después de una intervención (pre y postest), para examinar la variación del funcionamiento de la red del grupo de intervención y determinar el efecto del tratamiento mediante la reducción de la conexión de los síntomas más centrales de la red ${ }^{12}$. La estimación de redes según la condición clínica o sociodemográfica es importante para una mayor comprensión de la concurrencia interactiva de los componentes de los fenómenos clínicos y determinar el funcionamiento diferencial de estas redes ${ }^{13}$.

Otro estudio reciente evaluó dos redes de síntomas de ansiedad y depresión en dos tiempos: inicio del brote de la COVID-19 y posterior al umbral de contagio ${ }^{14}$. A su vez, dado que con este análisis también es posible estimar los cambios dinámicos de la red a través del tiempo, se ha evaluado longitudinalmente la dinámica de los estados de ánimo durante la primera cuarentena de COVID-19 en España ${ }^{15}$.

Así pues, la situación pandémica ha dado lugar a la aplicación de la metodología de redes en la evaluación de medidas clínicas de autoinforme, donde se reportan reacciones de miedo, ansiedad, depresión, estrés y amenaza por COVID-196-18. De igual modo, otra investigación recientemente evaluó el sistema de red de sintomatología depresiva en pacientes con hipertensión arterial ${ }^{19}$ en el contexto peruano. Sin embargo, urge una mayor divulgación y capacitación sobre este enfoque para su uso general en la investigación clínica sudamericana.
Como ejemplo, la Figura 1 representa un análisis de red a partir de los datos de un estudio publicado recientemente que desarrolló la medida Infectious Diseases Preventive Health Behavior Scale (ID$\mathrm{PHBS})^{20}$. En ella se pueden apreciar las correlaciones parciales de los 12 ítems de dicho instrumento (Figura 1).

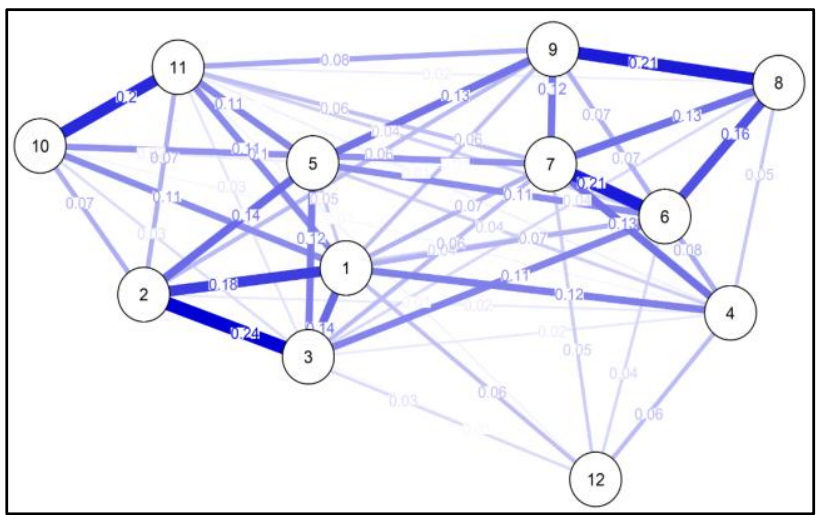

Figura 1. Análisis de red del ID-PHBS.

En conclusión, el análisis de red ofrece una valiosa contribución metodológica y práctica en las ciencias de la salud. De este modo, los profesionales clínicos cuentan con una herramienta para interpretar y traducir la información derivada de su investigación en una mejor atención integral en la población clínica de interés. Así, es posible enfocarse en una intervención concreta a partir de los resultados de red, desde la perspectiva de sistemas de modelos complejos $^{21}$. La evaluación de la red afianza una mayor explicación del funcionamiento de diversas condiciones de salud vinculadas al impacto de la COVID-19 y cómo sus mecanismos etiológicos interactúan entre sí.

\section{REFERENCIAS BIBLIOGRÁFICAS}

1. Scott J, Carrington PJ. The SAGE handbook of social network analysis. Los Angeles: SAGE; 2011. Disponible en: https://bok.lat/book/3364453/720e07.

2. Fried El, Van Borkulo CD, Cramer AO, Boschloo L, Schoevers RA, Borsboom D. Mental disorders as networks of problems: a review of recent insights. Soc Psychiatry Psychiatr Epidemiol. 2017; 52(1): 110. Doi: https://doi.org/10.1007/s00127-016-1319Z. 
3. Robinaugh DJ, Hoekstra RHA, Toner ER, Borsboom D. The network approach to psychopathology: a review of the literature 2008-2018 and an agenda for future research. Psychol. Med. 2020; 50(3): 35366.

Doi:

https://doi.org/10.1017/S0033291719003404.

4. Leme DEDC, Alves EVDC, Fattori A. Relationships Between Social, Physical, and Psychological Factors in Older Persons: Frailty as an Outcome in Network Analysis. J Am Med Dir Assoc. 2020; 21(9): 130915.e4.

Doi: https://doi.org/10.1016/j.jamda.2020.02.005.

5. Tosi G, Borsani C, Castiglioni S, Daini R, Franceschi $M$, Romano D. Complexity in neuropsychological assessments of cognitive impairment: A network analysis approach. Cortex. 2020; 124: 85-96. Doi: https://doi.org/10.1016/j.cortex.2019.11.004.

6. Hilland E, Landrø NI, Kraft B, Tamnes C, Fried E, Maglanoc $L$, et al. Exploring the links between specific depression symptoms and brain structure: $A$ network study. Psychiatry Clin Neurosci. 2020; 74(3): 220-1. Doi: https://doi.org/10.1111/pcn.12969.

7. Moriarity DP, Horn SR, Kautz MM, Haslbeck JM, Alloy LB. How handling extreme C-reactive protein (CRP) values and regularization influences CRP and depression criteria associations in network analyses. Brain Behav Immun. 2021; 91: 393-403. Doi: https://doi.org/10.1016/j.bbi.2020.10.020.

8. Isvoranu AM, Guloksuz S, Epskamp S, Van Os J, Borsboom D, GROUP Investigators. Toward incorporating genetic risk scores into symptom networks of psychosis. Psychol Med. 2020; 50(4): 636-43.

Doi:

https://doi.org/10.1017/S003329171900045X.

9. Fried El, Von Stockert S, Haslbeck JMB, Lamers F, Schoevers RA, Penninx BWJH. Using network analysis to examine links between individual depressive symptoms, inflammatory markers, and covariates. Psychol Med. 2020; 50(16): 2682-90. Doi: https://doi.org/10.1017/S0033291719002770.

10. Blanco I, Contreras A, Chaves C, Lopez-Gomez I, Hervas G, Vazquez C. Positive interventions in depression change the structure of well-being and psychological symptoms: A network analysis. J Posit Psychol. 2020; 15(5): 623-8. Doi: https://doi.org/10.1080/17439760.2020.1789696.

11. Ramos-Vera CA. Ramos-Vera CA. Métodos estadísticos modernos para evaluar diferencias en el contexto pandémico: El caso del género en el miedo a la COVID-19. Por: Ramos-Vera CA. Las redes de relación estadística en la investigación de nutrición. Nutr Hosp. 2021. 38(3):671-672. Disponible en: https://www.researchgate.net/publication/348440 703_Modern_Statistical_Methods_to_Assess_Diffe rences_in_the_Pandemic_Context_The_Case_of_G ender_in_Fear_of_COVID-

19_Metodos_estadisticos_modernos_para_evaluar _diferencias_en_el_contexto_pandemico_El_caso_

12. Wang Y, Hu Z, Feng Y, Wilson A, Chen R. Changes in network centrality of psychopathology symptoms between the COVID-19 outbreak and after peak. Mol Psychiatry. 2020; 25(12): 3140-9. Doi: https://doi.org/10.1038/s41380-020-00881-6.

13. Martín-Brufau R, Suso-Ribera C, Corbalán J. Emotion Network Analysis During COVID-19 Quarantine - A Longitudinal Study. Front Psychol. 2020; $\quad$ 11: e559572. Doi: https://doi.org/10.3389/fpsyg.2020.559572.

14. Ramos-Vera CA. Miedo a la COVID-19 en adultos peruanos mediante análisis de red. Rev Cubana Enferm. 2020; 36(1): e4082. Disponible en: http://www.revenfermeria.sld.cu/index.php/enf/ar ticle/view/4082.

15. Ramos-Vera CA. Estructura de red del impacto y la percepción de amenaza de la COVID-19. Rev Cubana Enferm. 2020; 36(1): e4136. Disponible en: http://www.revenfermeria.sld.cu/index.php/enf/ar ticle/view/4136.

16. Ramos-Vera CA. Red de síntomas psicopatológicos de la conducta alimentaria, depresión, ansiedad y estrés postraumático por la COVID-19. Rev cubana Enferm. De próxima aparición.

17. Ramos-Vera C, Baños-Chaparro, J, Ogundokun R. The network structure of depressive symptoms in 
Peruvian adults with arterial hypertension. F1000Research. 2021; 10: 19. Doi: https://doi.org/10.12688/f1000research.27422.1.

18. Peixoto EM, Palma B, Farias I, Santana N, Zanini D, Bueno JM. Questionário de Impulsividade, Autoagressão e Ideação Suicida para Adolescentes (QIAIS-A): propriedades psicométricas. Psicol Saúde Doenças. 2019; 20(2): 271-85. Doi: http://dx.doi.org/10.15309/19psd200201.

19. Ramos-Vera CA, Ramírez Y, Rojas E, Serpa A, García FA. Evidencias psicométricas mediante SEM y análisis de red de la Escala de Crecimiento Postraumático en adolescentes peruanos. Psicol Cond. De próxima aparición.

20. Ayandele $\mathrm{O}$, Kolawole SO, Ramos-Vera, C, Oguntayo R, Gandi J, Kator lorfa S, et al. The Infectious Diseases Preventive Health Behavior Scale (ID-PHBS): Development and Validation with an African Sample. 2020. Disponible en: https://ssrn.com/abstract=3776458.

21. Ramos-Vera CA. Conceptos de interés en la atención integral de los pacientes con multimorbilidad. Aten Primaria. 2021; 53(6). Disponible en: https://www.researchgate.net/publication/348249 395_Conceptos_de_interes_en_la_atencion_integr al_de_los_pacientes_con_multimorbilidad. 